Revue d'histoire de l'Amérique française

TEVUE D.HISTOIRE DE L'AMÉRIQUE FRANÇAISE

\title{
Les pratiques associatives et la constitution du champ de production littéraire au Québec (1760-1867)
}

\section{Pierre Rajotte}

Volume 45, numéro 4, printemps 1992

URI : https://id.erudit.org/iderudit/305017ar

DOI : https://doi.org/10.7202/305017ar

Aller au sommaire du numéro

\section{Éditeur(s)}

Institut d'histoire de l'Amérique française

ISSN

0035-2357 (imprimé)

1492-1383 (numérique)

Découvrir la revue

Citer cet article

Rajotte, P. (1992). Les pratiques associatives et la constitution du champ de production littéraire au Québec (1760-1867). Revue d'histoire de l'Amérique française, 45(4), 545-572. https://doi.org/10.7202/305017ar
Résumé de l'article

Le présent texte trace sommairement l'évolution des pratiques associatives " littéraires " au Québec de la Conquête en 1760 à la Confédération en 1867, et tente de déterminer de quel mode d'existence elles relèvent : celui d'une dépendance à l'endroit d'instances externes au champ littéraire (religion, politique, science, etc.) ou celui d'une autonomie fondée sur des déterminations proprement littéraires. L'auteur démontre entre autres que les pratiques associatives ont largement contribué à la constitution du champ de production intellectuelle, dont le champ littéraire est un sous-ensemble. Il constate en revanche qu'au sein des associations « formelles », la littérature est souvent soumise à des causes qui lui sont externes; ce qui va conduire certains lettrés à préférer les regroupements « informels ».
Tous droits réservés @ Institut d'histoire de l'Amérique française, 1992
Ce document est protégé par la loi sur le droit d'auteur. L'utilisation des services d'Érudit (y compris la reproduction) est assujettie à sa politique d'utilisation que vous pouvez consulter en ligne.

https://apropos.erudit.org/fr/usagers/politique-dutilisation/ 


\title{
LES PRATIQUES ASSOCIATIVES ET LA CONSTITUTION DU CHAMP DE PRODUCTION LITTÉRAIRE AU QUÉBEC $(1760-1867)^{1}$
}

\author{
PIERRE RAJOTTE \\ Centre de recherche en littérature québécoise \\ Université Laval
}

\section{RÉSUMÉ}

Le présent texte trace sommairement l'évolution des pratiques associatives «littéraires» au Québec de la Conquête en 1760 à la Confédération en 1867, et tente de déterminer de quel mode d'existence elles relèvent: celui d'une dépendance à l'endroit d'instances externes au champ littéraire (religion, politique, science, etc.) ou celui d'une autonomie fondée sur des déterminations proprement littéraires. L'auteur démontre entre autres que les pratiques associatives ont largement contribué à la constitution du champ de production intellectuelle, dont le champ littéraire est un sous-ensemble. Il constate en revanche qu'au sein des associations «formelles», la littérature est souvent soumise à des causes qui lui sont externes; ce qui va conduire certains lettrés à préférer les regroupements «informels».

\section{ABSTRACT}

This text summarily describes the evolution of the practices of literary associations in Quebec from the Conquest (1760) to Confederation (1867), and attempts to determine how they function: are these practices purely literary or are they dependent on other influences, such as religion, politics, science, etc. The author shows, among other things, that these practices contributed greatly to the establishment of an intellectual milieu, the literary field being part of this milieu. He establishes that in formal associations literature is influenced by external factors and that this situation will bring men of letters to prefer informal meetings.

1 Cet article est une version remaniée d'une communication présentée au $59^{\mathrm{c}}$ Congrès de l'ACFAS, en mai 1991, à l'Université de Sherbrooke. Quelques extraits en ont paru dans $L a$ vie littéraire au Québec, tome 1: 1764-1805 (Québec, Presses de l'Université Laval, 1991), 161180. Dans le cadre du projet de recherche sur l'«Histoire de la vie littéraire au Québec (17641914)», ont été dépouillés environ 75 périodiques, qui constituent la documentation de base de la présente étude; on en pourra consulter la liste au Centre de recherche en littérature québécoise de l'Université Laval.

RHAF, vol. 45, no 4, printemps 1992 
Les académies ont été un facteur décisif de l'autonomie du champ intellectuel et de sa division en branches de spécialités distinctes. Au sein de celui-ci, elles ont favorisé la suprématie du domaine littéraire. Elles ont donc joué un rôle capital dans la légitimation première de la littérature et de l'écrivain ${ }^{2}$.

Dans son étude Naissance de l'écrivain (1985), Alain Viala a mis au jour l'influence considérable des académies sur le processus d'institutionnalisation du champ littéraire en France au XVII ${ }^{\mathrm{e}}$ siècle. La formation d'un monde académique n'est pas le seul, mais certainement le plus manifeste des paramètres qui lui permettent d'observer la division du champ intellectuel en branches d'activités spécialisées où se sont forgés les traits distinctifs et les institutions du champ littéraire. Nous retenons de son étude que la question des regroupements intellectuels est significative puisqu'elle est à la base même du processus d'autonomisation et de légitimation de la littérature. Par définition, ce processus présuppose la constitution d'un système d'inter-relation dans lequel des individus, après avoir reçu un enseignement qui les préparait à la réception et, de façon plus limitée, à la production littéraire, sont portés, de par leurs intérêts communs, à se regrouper pour constituer un champ propice à leurs activités, fondé sur l'échange et la reconnaissance entre «pairs». Or en se regroupant, ils établissent les frontières qui les spécifient dans le champ intellectuel général et s'affranchissent de la tutelle d'autorités qui prétendent légiférer dans le domaine littéraire en fonction d'un pouvoir extérieur (politique, religion, etc.).

À titre de membre du groupe de recherche en «Histoire de la vie littéraire au Québec 1764-1914», qui vise précisément à cerner les processus de constitution et d'autonomisation de la littérature au Québec, nous avons tenté d'évaluer l'incidence des pratiques associatives sur le développement de l'activité littéraire au Québec au $\mathrm{XIX}^{\mathrm{e}}$ siècle. Ce travail a été entrepris à la suite d'un dépouillement systématique de plusieurs périodiques des $\mathrm{XVIII}^{\mathrm{e}}$ et $\mathrm{XIX}^{\mathrm{e}}$ siècles, et de différentes publications des associations. Les dimensions restreintes de cet article ne nous permettent toutefois pas de livrer en détail les résultats de notre recherche. Aussi nous limiterons-nous à tracer brièvement l'évolution des pratiques associatives de la Conquête en $\mathbf{1 7 6 0}$

2 Alain Viala, Naissance de l'écrivain. Sociologie de la littérature à l'âge classique (Paris, Éditions de Minuit, 1985), 50. 
à la Confédération en 1867, et d'évaluer sommairement leur apport dans la constitution du champ de production littéraire.

S'il fallait s'en tenir au nombre d'associations proprement littéraires, au sens où on l'entend aujourd'hui, la question serait bien vite réglée. Mais pour qui connaît l'ampleur du terme littéraire au XVIII ${ }^{e}$ et au $\mathrm{XIX}^{\mathrm{e}}$ siècle, la question reste complexe. Dans sa plus large extension, en effet, le terme peut désigner tout écrit de quelque nature qu'il soit, comme l'illustre bien Edmond Lareau dans son Histoire de la littérature canadienne (1874). Comme au XVIII ${ }^{e}$ siècle, les écrivains peuvent s'occuper de science autant que d'art et l'on conçoit la littérature comme le véhicule normal des connaissances. Évidemment la spécification des champs des autres disciplines telles que physique, géographie, etc., amènera par le fait même la littérature à spécifier son propre champ et à déterminer le littéraire par rapport au non littéraire. Mais il faudra quand même un certain temps avant que ne s'enclenche ce processus et surtout qu'il atteigne son but. La question qui se pose est précisément de savoir si les associations dites «littéraires» au XIX ${ }^{\mathrm{e}}$ siècle au Québec ont joué un rôle en ce sens. Théoriquement, le processus d'autonomisation s'engage à partir de l'indétermination pour évoluer vers la spécification. En principe, les associations d'abord très générales devraient normalement regrouper de plus en plus de gens unis par les mêmes intérêts. Certains de ces cercles de discussion devraient devenir des cercles spécifiquement littéraires, c'est-à-dire des cercles où le bien dire l'emporte sur les thèses. Qu'en est-il en réalité?

Au cours de ce travail, nous avons adopté une périodisation qui, pour politique qu'elle soit, n'en est pas moins représentative de l'évolution du champ de production littéraire à l'époque. Tant que ce dernier reste en voie de constitution, en effet, il demeure largement tributaire d'autres forces, et en particulier de la politique. La Conquête, l'Acte constitutionnel de 1791, le soutien du gouverneur, la présence du gouvernement à Québec, puis à Montréal (1840-1849), puis à Québec de nouveau (1860-1867), sont autant de facteurs qui influent sur son développement et, a fortiori, sur celui des pratiques associatives.

\section{LA CONQUÊTE}

La Conquête a largement favorisé le développement de groupes de pression et d'associations volontaires au pays. Il suffit d'étudier le Régime français pour s'en convaincre. Aucune forme de pratique associative n'y est sanctionnée, à l'exception des institutions tirant leur légitimité de la délégation royale (les hôpitaux généraux, la 
compagnie à charte, la manufacture royale, etc.), des confréries religieuses (comme la confrérie de la Sainte-Famille (1663), la confrérie de l'Adoration perpétuelle du Saint-Sacrement et de la bonne mort fondée à Montréal en 1732 par le sulpicien Antoine Déat) ou des sociétés commerciales ne donnant nullement lieu à ces regroupements formels et non lucratifs, à ces coalitions à la fois souples et durables d'individus que sont les associations.

En fait, en dehors de ceux qui visent à célébrer officiellement l'ordre établi, les rassemblements publics ne sont pas autorisés sous le Régime français. «Toute assemblée qui n'a pas eu au préalable la permission des autorités est tenue pour émeute; il en est de même pour toute délégation ou pour toute pétition: avant toute mise en mouvement, il faut obtenir l'accord de l'autorité ${ }^{3} »$. De là à dire que la voix du peuple ne peut se faire entendre, sinon que par les voies régulières de la hiérarchie, il n'y a qu'un pas. Qu'on en juge plutôt par les propos de Colbert qui répondait ainsi à Frontenac au sujet d'une assemblée des états:

Vous devez toujours suivre dans le gouvernement et la conduite de ce pays-là les formes qui se pratiquent ici, et que nos rois ont estimé du bien de leur service depuis longtemps de ne point assembler les états généraux de leur royaume, pour peut-être anéantir insensiblement cette forme ancienne, vous ne devez aussi donner que très rarement, et pour mieux dire jamais, cette forme au corps des habitants du dit pays; et il faudra même avec un peu de temps, et lorsque la colonie sera encore plus forte qu'elle n'est, supprimer insensiblement le syndic qui présente des requêtes au nom de tous les habitants, étant bon que chacun parle pour soi, et que personne ne parle pour tous ${ }^{4}$.

Bref les liaisons d'allégeance, de suzeraineté-vassalité qui caractérisent le Régime français amènent les individus à se référer à quelque chose, à quelqu'un qui se situe en dehors d'eux ou de leur seule volonté. Par conséquent, à quelques exceptions près, les institutions d'ancien régime sont moins des conjugaisons de volontés que des groupes de fait et des cadres contraignants et globaux de socialisation.

Il en va tout autrement au lendemain de la Conquête. Sous l'impulsion du dynamisme économique britannique, la régulation des rapports sociaux se fonde peu à peu sur une logique de production et

3 Marcel Trudel, Initiation à la Nouvelle-France. Histoire et institutions (Montréal et Toronto, Holt, Rinehart \& Winston, 1968), 169.

4 Cité par Gonzalve Doutre et Edmond Lareau, Histoire générale du droit canadien 1492-1791 (Montréal, Alphonse Doutre et Cie, libraires-éditeurs, 1872), 171. 
d'échange où des individus, porteurs de droits, créent un tissu complexe d'ententes réciproques du type contractuel, dans un cadre dominé par la propriété privée. La société est désormais conçue non plus comme une hiérarchie stable de statuts finement déterminés, propre à l'ordre féodal, mais comme une réunion volontaire d'individus libres et autonomes dont le principe de cohésion repose sur l'harmonie naturelle résultant de la poursuite libre et raisonnée des intérêts particuliers.

À cette logique de l'échange engendrée par le commerce britannique correspondent également un libre échange d'information et une plus grande communication entre l'État et les individus. Rappelons qu'en 1764 la première imprimerie et la première gazette officielle voient le jour dans la colonie. De plus, l'État est aux écoutes de différents secteurs de la population, notamment par les pétitions au roi qui se multiplient à partir de 1763. Enfin, on assiste au développement de regroupements volontaires d'individus unis par l'appartenance à une classe, à une corporation ou par des intérêts communs. Toutes apolitiques et privées qu'elles soient, certaines de ces associations volontaires côtoient bientôt la sphère publique réglementée par l'État, afin d'être en mesure de discuter avec elle des règles générales des échanges et des affaires. Qui plus est, elles ne tardent pas à agir comme une force instituante et à faire des pressions sur le gouvernement en se réclamant d'une nouvelle autorité, celle des arguments et du raisonnement public. En d'autres mots, elles deviennent bientôt le lieu d'orchestration d'une sphère publique politique qui s'exerce par la persuasion rhétorique et l'éloquence, soit les éléments constituants des pratiques constitutives de l'opinion publique au sens défini par Jürgen Habermas 5 .

\section{LES PREMIÈRES ASSOCIATIONS BRITANNIQUES}

Dès leur arrivée, les Britanniques fondent des associations de toutes sortes en particulier des loges maçonniques, des sociétés de convivialité (clubs), quelques sociétés de débats (free debate), puis au $\mathrm{XIX}^{\mathrm{e}}$ siècle quelques sociétés savantes et sociétés d'artisans (mechanics' institutes). Déjà en 1759 , presque tous les régiments anglais ont leurs loges maçonniques dites militaires ${ }^{6}$. Les marchands anglais qui

5 Jürgen Habermas, L'espace public. Archéologie de la publicité comme dimension constitutive de la société bourgeoise (Paris, Payot, 1978), 324 p.

6 Ces loges sont fondées au Canada dès l'arrivée des troupes de Wolfe et d'Amherst. Dans son Journal, le capitaine Knox note en date du 27 décembre 1759: "The anniversary of Saint John the Evangelist was duly observed by the several lodges of free masons in the garrison.» John $\mathrm{H}$. Graham a relevé sept loges franc-maçonnes militaires dans les garnisons de Québec et sept dans les garnisons de Montréal. John H. Graham, Outlines of the History of Free Masonry in the Province of Quebec (Montréal, John Lovell, 1892), 36-37. 
suivent l'armée et les membres du nouveau personnel administratif ne tardent pas à établir eux aussi des loges comme ils en ont connues en Angleterre ou à New York. En 1760, des marchands de Québec fondent la loge St.Andrew. Des marchands de Montréal les imitent, en 1761, avec la loge St.Peter. De 1759 à 1790, pas moins d'une trentaine de loges sont fondées dans la colonie, ce qui est d'autant plus considérable que les Anglais y sont encore peu nombreux.

La franc-maçonnerie introduite au Québec par les régiments britanniques établit une certaine continuité avec la sphère publique représentative dont l'État britannique est le support. Parmi leurs membres, plusieurs loges comptent des gouverneurs, Guy Carleton par exemple, des lieutenants-gouverneurs, des secrétaires d'État, des fonctionnaires, de riches marchands, des militaires haut gradés, etc. Ces loges se distinguent par leur principe de cohésion, soit la poursuite libre et raisonnée d'intérêts particuliers. Certaines par exemple ressemblent à de véritables cours au sein desquelles les classes moyennes marchandes cherchent à obtenir les faveurs des représentants du roi. D'autres, composées presque exclusivement de marchands réunis afin de discuter des moyens de favoriser leurs intérêts communs et de faire pression sur la sphère publique réglementée par l'État, s'emploient à faire publier des chansons, des sermons, à organiser des soirées théâtrales, à rédiger des pétitions sur la question des droits et privilèges, sur l'établissement d'une Chambre d'assemblée. En comparant des listes de membres dirigeants de certaines loges de 1760 à $1790^{7}$ et des listes de pétitionnaires ${ }^{8}$ pour la même période, on retrouve presque les mêmes noms ${ }^{9}$. De 1763 à 1790, ces pétitions seront l'une des premières manifestations de certaines forces sociales qui veulent être en mesure d'influencer les décisions du pouvoir en faisant appel à «l'usage public de l'argumentation».

Les Anglais se réunissent également en sociétés de convivialité et en sociétés de «débats libres». Les premières sont des cercles restreints dont les membres se rencontrent à des intervalles plus ou moins réguliers pour deviser, tout en festoyant, de questions et d'intérêts qui leur sont communs. L'une des premières associations du genre, la

7 John H. Graham, op. cit., 36-83.

8 Arthur Doughty et Adam Shortt, dir., Documents relatifs à l' histoire constitutionnelle du Canada 1759-1791 (Ottawa, Imprimeur du roi, $2^{c}$ édition, 1921), passim.

9 Mentionnons entre autres les noms de Alexander Fraser, Thomas McCord, John Ross, John Saul, Alexander Lawson, Francis Anderson, William Fraser, John Grant, Alexander Campbell, William Webb, James Gibbons, C. J. Tanswell, James Creig, John Munro, John Fraser, Lauch Smith, Thomas Aylwin, John Aitkin, John Lynd, John Chisholm, Peter Mills, etc. 
Select Society (1774), témoigne de l'intérêt grandissant pour la discussion publique.

Hier la Société choisie (qui a été instituée dans cette ville [Montréal], il y a environ un an, pour conférer librement touchant des matières choisies) tint son assemblée annuelle à la Racetaverne. Les principaux officiers militaires, de la ville et des environs, furent invités à diner [...]. Ensuite un des membres prononça une harangue convenable à la nature de la Société, démontrant les avantages résultants d'une fréquente communication littéraire ${ }^{10}$.

Toutefois, les plaisirs de la table prennent, dans ces sociétés, une place au moins aussi importante que celle de la discussion. C'est le cas des Beaver club (1785), Beef-Steak club (1792) et Greybeards club (1794), etc.

En revanche, les sociétés de débats, comme la Minerva Free Debating Society (1777) et la Robin Hood Society (1791) font de la discussion l'objet principal de leurs réunions. Leurs membres se réunissent régulièrement, voire chaque semaine, pour discuter le pour et le contre d'une question annoncée au préalable. Loin d'être réduit à une lutte où des thèses opposées sont défendues par leurs partisans respectifs, le débat vise à susciter des opinions pour les soumettre à la discussion. Présumés de même poids pour tous, les arguments sont disposés comme sur les plateaux d'une balance. Les opinions retenues, c'est-à-dire les plus valables, sont celles qui résistent à la discussion et mènent à une conclusion unanimement admise. Il s'agit donc de s'éclairer mutuellement et de favoriser l'exercice pratique de l'éloquence plutôt que de défendre une vérité intangible. D'ailleurs, pour éviter l'esprit de parti et les abus qu'il entraîne, on n'hésite pas à privilégier des sujets ne menant à aucune conclusion pratique. Le 25 janvier 1777 par exemple, la Minerva Free Debating Society soumet à ses membres: «I. Whether the common Observation, That «a reformed Rake makes the best Husband», be true or not? II. Whether the [first] Declarations of Love upon honourable Terms be consistent with this Modesty and Reserve of the Fair-sex ${ }^{11} »$ ? La semaine suivante, la Gazette de Québec rapportait les résulats de la discussion et annonçait les questions qui seraient discutées lors de la prochaine réunion:

Mr. Printer, As a Lover of literary Intelligence, even of the Smallest Consequence, I have Hopes you will insert the

10 La Gazette de Québec, 536 (20 avril 1775): 3.

11 La Gazette de Québec, 595 (23 janvier 1777): 3. 
following Resolutions (to the Questions publish'd in your last) formed by the united Sentiments of a Majority in the Minerva Free Debating Society.

I. Resolv'd, That the common Observation, that «a reform'd Rake makes the best Husband,» is true.

II. Resolv'd, That the first Declarations of Love upon honourable Terms are unconsistent with the Modesty and Reserve of Fairsex.

Le $1^{\text {er }}$ février 1777 :

I. Whether Duelling be of Advantage or Disadvantage to Society? II. Whether Shakespear, the admired dramatic Author, would have shone more conspicuous in the literary World, had he receiv'd the boasted Advantages of a classical Education ${ }^{12}$ ?

Enfin, au début du $\mathrm{XIX}^{\mathrm{e}}$ siècle, la bourgeoisie anglophone fonde des sociétés d'artisans comme le Montreal Mechanics' Institutes ${ }^{13}$ (1828) et le Quebec Mechanics' Institute (1830), de même que des sociétés savantes comme la Quebec Literary and Historical Society (1824) et la Montreal Natural History Society (1825). Conçues dans un contexte de promotion de l'Empire britannique en Amérique, ces dernières, qui empruntent beaucoup aux modèles britanniques des Literary, Scientific and Philosophical Societies, partagent par leurs activités - conférences publiques, bibliothèque publique, musée, concours ouverts au public — une même volonté de s'approprier «scientifiquement» le milieu colonial en favorisant «par tous les moyens possibles la découverte, la collection et l'acquisition de toutes les informations tendant à répandre du jour sur l'histoire naturelle, civile et littéraire de l'origine des Provinces britanniques dans l'Amérique septentrionale ${ }^{14} \gg$. Au demeurant, dans une société où l'information fonde de plus en plus le pouvoir, il importe d'accumuler l'une pour s'assurer l'autre. À la conquête militaire, succède donc celle du savoir. Dans une perspective visant à établir les avantages de l'anglicisation

12 La Gazette de Québec, 596 (30 janvier 1777): 4.

13 D'origines anglaise et écossaise, les Mechanics' Institutes ont pour objectif de favoriser «the diffusion of useful knowledge» parmi les artisans et les travailleurs. Il s'agit de l'une des premières formes d'éducation des adultes, rendue nécessaire par la révolution industrielle. Voir à ce sujet Pierre Leduc, «Note sur les origines et la première phase de développement du mouvement des Mechanics' Institutions en Grande-Bretagne», Recherches sociographiques, 16,2 (mai-août 1975): 249-260; Nora Robins, «The Montreal Mechanics' Institute: 1828-1870», Canadian Library Journal, 38,6 (décembre 1981): 373-379.

14 Louis-P. Turcotte, «Histoire de la Société littéraire et historique depuis sa fondation», Transactions of the Literary and Historical Society of Quebec, New Series, XIII (Sessions of 1877-78-79), 25. 
de la colonie, il s'agit d'acquérir et de sélectionner l'information de façon à rendre compte de la supériorité du régime britannique dans l'Ámérique septentrionale. Citons le cas de la Literary and Historical Society of Quebec qui, pendant plusieurs années consécutives, offre une dizaine de prix entre autres pour:

1. Une partie quelconque de L'HISTOIRE NATURELLE, le sujet ayant rapport à l'Amérique Septentrionale Britannique.

2. Une partie quelconque de L'HISTOIRE DU CANADA ou ses antiquités, ou sur le langage et les mœurs des Aborigènes.

3. Le meilleur poème sur un sujet ayant rapport à L'AMÉRIQUE SEPTENTRIONALE Britannique.

4. Un bon essai historique, tendant à remplir la lacune qui existe dans L'HISTOIRE DU CANADA, à dater de l'époque où Charlevoix termine son histoire en l'an $1749^{15}$.

5. Pour le meilleur écrit sur aucun sujet lié aux Sciences.

6. Un sujet ayant rapport aux ARTS UTILES, et particulièrement ceux qui peuvent être applicables à l'Amérique Septentrionale Britannique $^{16}$.

Les modèles d'associations britanniques se sont donc rapidement répandus au pays. Mais quelle influence ont-ils exercée sur le comportement associatif des Canadiens français?

\section{LES PREMIÈRES ASSOCIATIONS FRANCOPHONES}

$\mathrm{Au}$ lendemain de la Conquête, les Canadiens (Canadiens français) s'initient rapidement à l'art d'exercer des pressions sur le pouvoir en envoyant, à l'instar des sujets britanniques, des pétitions à Londres. Mais ils forment très peu d'associations proprement dites. C'est que défavorisés économiquement et politiquement, ils sont moins sensibles aux motivations qui poussent les Britanniques à se regrouper. Les marchands anglais, par exemple, s'unissent avant tout pour mieux défendre leurs intérêts particuliers rattachés au commerce. Or très peu de francophones ${ }^{17} s^{\prime}$ intègrent au nouvel ordre commercial sous le

15 À noter que la période étudiée par Charlevoix se termine en 1732 et non en 1749.

16 Voir Le Canadien, 31 août 1836, 2; 4 août 1837, 1; 24 août 1838, 3; 22 juillet 1839, 2.

17 Citons le cas du jeune négociant canadien Pierre Gamelin, marguillier de sa paroisse, qui se joignit à une loge franc-maçonne anglaise et non catholique. Ses nouveaux liens commerciaux avec les Anglais le poussaient en ce sens. Étienne Montgolfier, grand vicaire, qui jugea incompatible la fonction de marguillier et celle de franc-maçon, le pria de choisir entre les deux allégeances. «Lettre d'Étienne Montgolfier à l'évêque de Québec, 20 janvier 1771», cité par Jean-Paul de Lagrave, Fleury Mesplet (1734-1794). Diffuseur des lumières au Québec (Montréal, Patenaude Éditeur, 1985), 444-445. 
régime anglais. D'où leur peu d'enthousiasme pour ce type d'association.

Malgré tout, les Canadiens profitent dans une certaine mesure des possibilités que leur offre le nouveau régime. Aiguillonnés par l'exemple des marchands anglais, ils vont chercher à adapter les pratiques associatives à leurs besoins. Ainsi, à défaut de «bien particulier» à défendre, on se rabat sur le «bien commun», en cherchant entre autres à combattre l'ignorance et à défendre les intérêts de la nation canadienne-française. À la dimension commerciale on préfêre la dimension philosophique et idéologique. En cela, les Canadiens français tendent à reproduire le modèle associatif français. Leurs premières associations sont d'ailleurs le fruit de quelques esprits révolutionnaires français - Valentin Jautard, Fleury Mesplet, Henri Mézière - qui poursuivent en Amérique le combat des Lumières. Notons cependant que dans l'ensemble, ces associations sont passablement éphémères. Il y a lieu, la plupart du temps, de parler d'épiphénomènes. C'est le cas entre autres du projet de l'Académie de Montréal en 1778, de l'association franc-maçonne les Frères du Canada fondée en 1786, de la Société des Patriotes en 1790, de la Société des débats libres en 1791.

Dans l'ensemble, le but de ces associations est de promouvoir la discussion et l'échange d'information. L'insistance avec laquelle on réaffirme leur rôle didactique est constante. Elle a quasi valeur de leitmotiv. Le faire-part qui annonce l'Académie de Montréal par exemple la définit essentiellement comme un lieu d'information et de formation pour les académiciens eux-mêmes, certes, mais aussi pour une population jusqu'alors maintenue dans l'ignorance. «Notre corps, précise le secrétaire, se doit à lui-même, et encore plus à la société. Le désir de nous instruire est inséparable du soin d'instruire les autres. [...] Nous aimons les sciences, il faut donc, suivant notre institution, encourager les émules ${ }^{18} . »$

Toutes éphémères qu'elles soient, ces pratiques associatives constituent des tentatives non négligeables pour organiser et valoriser le champ intellectuel, dont la littérature est un sous-ensemble. En effet, si la plupart des associations britanniques sont réservées à une élite marchande ou militaire désireuse de se distinguer des autres groupes sociaux par ses richesses ou sa condition sociale, celles des francophones réunissent une petite bourgeoisie qui tente de plus en plus de se définir en fonction de sa culture et de sa compétence intellectuelle. $\mathrm{Au}$ sein de ces associations, la discussion n'est plus uniquement 
l'affaire d'un rang social, mais de tout homme capable de raisonner. Ainsi, bien que l'Académie de Montréal n'explique jamais clairement quels sont ses critères d'admission, la lettre dans laquelle «le Canadien curieux» - pseudonyme attribué à Pierre-Louis Panet - et «le Sincère» posent leur double candidature ne laisse aucun doute sur l'importance de connaître les «principes de Montesquieu, Voltaire et Raynal et autres auteurs qui font honneur à l'humanité ${ }^{19}$ », que cette connaissance soit réelle ou prétendue.

Dans un sens, ces associations tendent à promouvoir une sorte d'égalité, une parité sur la base de laquelle seule l'autorité des arguments peut s'imposer. Fondée en 1791, la Société des débats libres de Montréal tend précisément à reproduire sur le plan proprement intellectuel la relation qui démarque sur le plan économique les propriétaires des non-propriétaires, et sur le plan social, les nobles des non nobles. Une polémique au sujet de la société l'illustre d'ailleurs on ne peut mieux.

Le 29 septembre 1791, dans une lettre ouverte publiée dans la Gazette de Montréal, un citoyen de Boucherville, François Vassal de Monviel, qui appartient à la noblesse canadienne, reproche à la Société des débats de discuter de sujets insignifiants et l'invite à choisir des thèmes de réflexion plus profitables à la collectivité. Cet écrit a l'effet d'une traînée de poudre, et suscite, la semaine suivante, un tollé de protestations dans la Gazette. Le 6 octobre, trois des quatre pages du journal sont remplies de lettres -10 lettres en français et 2 en anglais - conspuant le ton réprobateur de Monviel. À y regarder de près, on s'aperçoit que la polémique cache une confrontation entre d'une part, une noblesse qui n'admet pas que des individus puissent débattre publiquement de sujets divers au nom de la seule valeur de leurs idées, et, d'autre part, une bourgeoisie qui table sur une légitimité proprement rationnelle. Pour les membres de la Société des débats, le raisonnement public s'exerce non pas en fonction du rituel des hiérarchies, mais en fonction de la compétence de chaque individu. À leurs yeux, leur cercle constitue une enclave privilégiée où règne une égalité indépendante des diverses conditions sociales. C'est au nom de cette égalité qu'ils ripostent massivement à Vassal de Monviel. La réponse de l'un de ceux-ci est d'ailleurs sans équivoque:

Monsr. Mesplet, la Société des Débats libres a eu en vue dans son institution l'égalité primitive: je vous prie d'apprendre ceci par la voie de votre feuille à Mr. Vassal se stylant de Monviel, et de l'informer que la Société ne reconnaissant point ces antiques

19 La Gazette littéraire, 4 novembre 1878, 84. 
fatras de Gentilhommerie qui éblouirent nos bénêts d'Ancêtres, je ne puis l'écouter ni lui répondre en la qualité de Gentilhomme. Qu'il reparaisse sur la Scène dans cette égalité primitive, à la bonne heure: je serai alors du Parterre et j'en remplirai l'office avec plaisir ${ }^{20}$.

Cette opposition entre des individus qui axent leur intervention en fonction de leur savoir et ceux qui se réclament de leur condition sociale est l'indice qu'un premier champ intellectuel canadien tend à s'organiser, grâce en partie aux associations. Mais la nouvelle constitution de 1791 détourne passablement le sens de ce courant au profit de la politique.

\section{LE TOURNANT POLITIQUE: L'ACTE CONSTITUTIONNEL DE 1791}

Jusqu'à la nouvelle Constitution de 1791, les quelques pratiques associatives des Canadiens avaient constitué un pis-aller à leur exclusion de l'économie et de la politique. Il en va tout autrement à partir du moment où ils ont accès à la Chambre d'assemblée. Dès lors ils y concentrent toutes leurs forces au détriment des autres formes d'association qui leur paraissent moins efficaces. Certes, pour s'initier au régime parlementaire, on fonde, le 14 juin 1792, le Club constitutionnel, mais cette initiative demeure un cas isolé et éphémère. Une fois qu'ils ont saisi le fonctionnement et les avantages de la Chambre d'assemblée, les Canadiens y vouent un culte presque absolu.

Ce sur-investissement n'est pas sans conséquence sur les tentatives de regroupements littéraires, comme en témoigne le projet avorté d'une société littéraire à Montréal en 1817. Dans l'Aurore du 11 août 1817 , un correspondant qui signe «Philomathas» fait écho à un autre correspondant «Studiosus» qui déplorait une semaine plus tôt que ni Québec ni Montréal ne possédât de société littéraire. À ses yeux, une telle société représenterait «un excellent moyen pour donner l'émulation, porter au travail, et faire vaincre la paresse naturelle de l'esprit ${ }^{21} »$, mais qui plus est, elle contribuerait à «rendre le nom Canadien illustre dans la littérature». En fait, «Philomathas» voit dans le modèle européen, un gage de succès pour les lettres canadiennes. Puisque le développement des académies et des sociétés littéraires a favorisé la production en Europe, pourquoi en serait-il autrement au Canada?

On sait que les Académies et autres sociétés littéraires et scientifiques, n'ont commencé à s'établir en Europe qu'à la fin du

20 Un membre, la Gazette de Montréal, 51 (6 octobre 1791): 2.

21 «Société littéraire de Montréal», L'Aurore, 1,23 (11 août 1817): 1. 
$16^{\text {ème }}$, ou au commencement du $17^{\text {ème }}$ siècle, et que ce n'est que depuis ce tems qu'il a paru des ouvrages écrits avec goût, avec pureté et avec délicatesse. Les découvertes brillantes, les inventions utiles dans les arts et dans les sciences sont presque toutes postérieures à cette époque.

En établissant ainsi un rapport de cause à effet, «Philomathas» vise à faire admettre que «si le Canada n'a produit jusqu'à ce jour aucun savant, aucun littérateur distingué, c'est sans doute au défaut d'institutions semblables qu'il faut l'attribuer». Son projet n'aura cependant pas de suite. Tout au plus provoque-t-il quelques réactions dans l'Aurore. Le 16 août, un correspondant répond à ceux qui parlent «d'institutions et de Sociétés littéraires et scientifiques, [...] comme si nous n'avions rien de semblables»:

N'avons-nous pas une Société d'agriculture à Québec, une institution de la Vaccine, une Société de Fleuristes à Montréal? Nous avons aussi une Société politique et économique. Qui estelle, me demandera-t-on? C'est notre Chambre d'Assemblée. À mon avis, cette institution vaut bien une Académie des Sciences et une Société littéraire ${ }^{22}$.

Une semaine plus tard, «Studiosus» reproche à ce dernier d' «ignorer ce que c'est qu'une Académie ou une Société littéraire». Selon lui, l'Institution de la Vaccine, la Société d'Agriculture et «à plus forte raison celle des Fleuristes» ne sont pas des sociétés littéraires. Quant à la Chambre d'assemblée, il reconnaît son importance, mais estime que loin de nuire à son bon fonctionnement, une société littéraire aurait plutôt pour effet de l'améliorer: «Pourquoi donc regardez-vous comme inutiles les meilleurs moyens d'acquérir des connaissances, et les talents de parler avec facilité? Des assemblées où l'on discuterait sur des sujets scientifiques et littéraires ne seraient-elles pas de bonnes écoles pour préparer aux discussions politiques et économiques ${ }^{23}{ }^{2}$ ? Visiblement, pour faire admettre l'idée d'une société littéraire, ses promoteurs doivent insister sur les avantages politiques qu'en retireraient les Canadiens. De plus, force est de reconnaître que la Chambre d'assemblée demeure dans l'esprit de plusieurs l'association par excellence pour faire progresser la cause canadienne. D'où l'intérêt presque exclusif qu'on lui accorde, du moins jusqu'aux années 1830.

Cet intérêt des Canadiens pour une institution politique légitimée tient sans doute à l'accès inespéré au pouvoir qu'ils y trouvent. Mais les soupçons de l'autorité coloniale à l'endroit de toute autre tentative

22 Un campagnard, L'Aurore, 1,24 (16 août 1817): 1.

23 Studiosus, $L^{\prime}$ Aurore, 1,26 (30 août 1817): 2. 
d'association ne leur laisse guère le choix. En effet, l'opposition manifestée à la Chambre d'assemblée par les Canadiens amène l'autorité coloniale à soupçonner ces derniers, voire à les accuser de déloyauté, sinon de trahison. Dans ce contexte, toute forme de regroupement est rapidement suspecté de conspiration: «association et conspiration sont une seule et même chose ${ }^{24}$ ». La disparition de la Société littéraire de Québec quelques mois après sa fondation en 1809 l'illustre bien. Pour inaugurer les activités publiques de la Société, les membres s'empressent de témoigner leur loyauté à la couronne britannique en organisant un concours de poésie consacré à la célébration de la naissance de George III. Que ce soit par les textes primés, mais plus encore par les discours de Louis Plamondon et de François Romain qui précèdent la remise des prix en juin 1809, l'apologie du monarque anglais n'a d'égale que le factum contre la Révolution française et contre Napoléon, «homme né pour la terreur du genre humain qui a ravagé toutes les nations ${ }^{25}$ ». Louis Plamondon en particulier se livre à un véritable panégyrique de la Conquête: «Bénissons à jamais l'heureux moment où les armes victorieuses de l'Angleterre, sous l'immortel Wolfe, nous rendirent sujets de l'Empire Britannique» (p. 12). À la fin de son discours, il bénit le ciel d'avoir fait passer le Canada à l'Angleterre et de lui avoir épargné les horreurs de la Révolution. Il prie même qu'on admire pour sa fermeté, pour sa valeur et son savoir, ses talents et son mérite, le gourverneur Craig, celui-là même qui, quinze jours auparavant, le 15 mai 1809 , avait fait contre la majorité populaire de la Chambre d'assemblée législative un petit coup d'État en proclamant la dissolution du parlement.

En dépit de ce loyalisme dithyrambique dont se réclame la société, le Quebec Mercury ne tarde pas à la juger «useless, if not prejudicial ${ }^{26}{ }_{\Downarrow}$. En fait, au moment où s'intensifie la lutte politique entre le Parti canadien et le gouvernement colonial britannique, la Société littéraire peut difficilement poursuivre ses activités sans risquer d'être suspectée de trahison par le premier ou de conspiration par le second. $\mathrm{Au}$ risque d'être prise à partie comme les parlementaires et les journaux canadiens-français (en particulier le Canadien), bref tout ce qui d'une manière ou d'une autre compromet le projet d'anglicisation du régime colonial, elle disparaît donc quelques mois seulement après sa fondation. Poser la question en ces termes, c'est dire que seules les associations vouées explicitement ou implicitement à ce projet ont

24 La Minerve, 21 avril 1834, 2.

25 Louis Plamondon, «Discours prononcé devant la Société littéraire de Québec», Séance de la Société littéraire de Québec, tenue samedi le 3 juin 1809. Floreamus in memoribus (Québec, Imprimée par ordre de la Société par J. Neilson, imprimeur-libraire, 1809), 11.

26 A Smoker and News Paper Reader, The Quebec Mercury, 5,28 (10 juillet 1809): 217. 
droit de cité. Les Associations loyales, qui se multiplient pendant les premières décennies du $\mathrm{XIX}^{\mathrm{e}}$ siècle, en font foi, de même que plusieurs sociétés fraternelles et de charité formées pour que «les préjugés qu'un peuple descendu de nations différentes et rivales entretient naturellement l'un contre l'autre se dissipent graduellement ${ }^{27}{ }_{\text {». C'est }}$ également le cas des sociétés savantes qui, à partir d'un travail commun sur un savoir «neutre», poursuivent un travail d'intégration des élites lettrées. En fait, l'existence de ces sociétés dépend en grande partie des subsides que leur accordent les gouvernants. Aussi, dès qu'elles tentent de s'émanciper de leur tutelle, se condamnent-elles à disparaître, comme tend à le prouver la fusion de la Société pour l'encouragement des sciences et des arts (1827) avec la Literary and Historical Society of Quebec (1824).

Les sociétés savantes se répandent au pays à partir des années 1820 , en particulier au sein du milieu anglophone. La plus célèbre, qui existe encore aujourd'hui, est la Literary and Historical Society of Quebec (LHSQ). Fondée en 1824 par le gouverneur Lord Dalhousie dans le but de favoriser la découverte de toute information, tant historique que scientifique, au sujet de la colonie, elle ne tarde pas à susciter la méfiance des Canadiens français. Le 17 avril 1824, trois mois après sa fondation, le Spectateur canadien exprime des réserves quant aux objectifs de la société qui risque de mener à une «histoire par autorité» ayant pour fin de «canoniser les mesures de l'administration». Dans Le Canadien, certains mettent également en doute qu'«un Gouverneur, un Lieutenant-Gouverneur, un juge en chef [puissent] écrire l'histoire d'un pays qu'ils gouvernent» de façon impartiale et objective.

Quel écrivain, en effet, oserait s'élever contre une autorité semblable? ce ne serait que l'autorité d'un simple individu contre celle de tout un corps. [...] c'est que rien ne sera vrai et bon, que ce qui viendra par la voie de cette Société. Ce sera l'histoire des «Gouverneurs publiée par autorité, dans le siège du Gouvernement et par les Nourrissons du Gouvernement, présidés par les Chefs du Gouvernement». Quel poids n'aura pas une telle histoire! Tout le reste sera mensonger et ne vaudra pas la peine d'être écouté, ni au dedans ni au dehors ${ }^{28}$.

N'appréciant guère le parti-pris et le caractère exclusif de la nouvelle société qui limite le nombre de ses membres en exigeant une

27 Cité par John Hare et Jean-Pierre Wallot, Les imprimés dans le Bas-Canada 18011810 (Montréal, Presses de l'Université de Montréal, 1967), 92.

28 Le Canadien, 5,11 (31 mars 1824): 2. 
admission de $5 \mathfrak{-}$ - soit «le prix d'un bon microscope ${ }^{29}$ » -, et une cotisation annuelle de $3 £$, quelques jeunes Canadiens et une poignée de Britanniques décident donc, au printemps de 1827, de marcher sur ses brisées en fondant à Québec la Société pour l'encouragement des sciences et des arts au Canada. En exigeant une cotisation d'une guinée par année seulement, celle-ci peut bientôt compter sur plus de membres que sa rivale. Son succès n'est pas sans alarmer le gouverneur James Kempt qui intervient alors en faveur de leur fusionnement. Il parvient à ses fins à l'été de 1829 , alors que les membres des deux sociétés acceptent de se regrouper au sein de la plus ancienne.

Cette fusion s'inscrit-elle dans la politique de Frederick Haldimand, «qui avait voulu, par l'établissement d'une bibliothèque bilingue, en 1779, unir le plus possible les gens des deux races dans un organisme culturel, pour leur donner une mentalité commune et conforme aux fins de la politique anglaise ${ }^{30} »$ ? Ou, plus simplement, en 1829 une société unique apparut-elle plus viable que deux poursuivant des buts identiques dans une même ville? Quoi qu'il en soit, la fusion ne semble pas avoir très bien réussi aux Canadiens de langue française, qui participent peu à l'activité de la nouvelle société. Deux ans après l'union, seulement 29 membres sur 126 (23\%) sont de langue française. De plus, bien que quatre documents historiques de langue française y soient publiés de 1838 à 1861, les réunions mensuelles se déroulent en anglais (tous les rapports sont en anglais), les publications régulières et les conférences également. De 1829 à 1867, on publie dans les Transactions de la LHSQ 127 articles, dont trois seulement en français. Enfin, «les Canadiens français occupent environ $14 \%$ des sièges au conseil d'administration, pourcentage inférieur à celui de leur participation numérique réelle ${ }^{31} »$. Bref, même si elle se veut représentative des deux groupes ethniques à Québec, la LHSQ privilégie la culture anglophone. On observe d'ailleurs la même tendance au sein de la plupart des sociétés savantes ou d'artisans de l'époque.

Ainsi, le nouveau régime parlementaire dans lequel les Canadiens français sur-investissent, de même que le soupçon de l'autorité coloniale qui diffère toute forme de regroupement potentiellement non «loyale», entravent les rares projets de sociétés littéraires. Malgré tout,

29 Luc Chartrand, Raymond Duchesne et Yves Gingras, Histoire des sciences au Québec (Montréal, Boréal, 1987), 80.

30 Antonio Drolet, Les bibliothèque canadiennes (1604-1960) (Montréal, Le Cercle du livre de France, 1965), 115.

31 Ginette Bernatchez, «La Société littéraire et historique de Québec (The Literary and Historical Society of Quebec), 1824-1890», Revue d'histoire de l'Amérique française, 35,2 (septembre 1981): 185. 
ces tentatives sont significatives car elles marquent une certaine évolution du champ littéraire qui relève désormais de deux conceptions. La première consiste à s'intéresser à toutes sortes de sujets, la seconde procède d'une division du champ intellectuel en branche d'activités spécialisées. La Société littéraire de Québec qui à ses débuts tient «deux assemblées publiques par mois; où chacun [des membres], ou du moins plusieurs d'eux ont des sujets à discuter» sur des questions les plus diverses - dans Le Canadien du 29 avril 1809, l'un d'entre eux rapporte que la Société avait accepté une conférence et une discussion sur le sujet: «Si la musique[a] un effet médicinal»-, participe de la première. De même, pour faire admettre l'idée d'une société littéraire à Montréal en 1817, «Philomathas» table sur l'acception traditionnelle de la notion de littérature, conçue comme le véhicule normal des connaissances tant scientifiques qu'artistiques et littéraires.

Il serait à souhaiter qu'il y eût dans la Société, des membres capables de donner aux autres des notions claires et précises, sur les sciences les plus utiles, dont on a plus souvent occasion de parler dans la société en général, et sans la teinture desquelles il est impossible de réussir en littérature; telles que l'histoire naturelle, la Chymie, la Botanique, l'Anatomie, la Physique et 1'Astronomie ${ }^{32}$.

Reconnue par la tradition humaniste, cette conception qui privilégie un savoir de type encyclopédique demeure en grande partie à la base des sociétés savantes de l'époque, où au cours d'une même réunion, il peut être question de sujets scientifiques, historiques, philosophiques, littéraires, etc ${ }^{33}$. Apparaît cependant au sein de ces sociétés un souci de distinction entre les divers domaines intellectuels. «Dans le but de procurer à chacun des membres l'occasion de suivre avec plus d'avantage le genre d'étude auquel il désirerait donner une attention plus particulière ${ }^{34} \gg$, la plupart des sociétés savantes se divisent en trois ou quatre sections qui généralement tiennent leurs séances et délibèrent séparément. À la LHSQ par exemple, on distingue la classe d'histoire naturelle, la classe des arts, la classe des sciences et la classe

32 «Société littéraire de Montréal», L'Aurore, 1,23 (11 août 1817): 1-2.

33 Un soir de mars 1829 , on se réunit à la Société pour l'encouragement des sciences et des arts pour récompenser les auteurs de travaux scientifiques, entendre une conférence sur «La philosophie du goût», donnée par le révérend George Bourne, et enfin admirer quelques tableaux de Joseph Légaré, maître de la peinture canadienne de l'époque. Le compte rendu de cette soirée est donné dans le Star and Commercial Advertiser de Québec, le 25 mars 1829; cité par Luc Chartrand, Raymond Duchesne et Yves Gingras, op. cit., 82.

34 Règles et ordres de la Société pour l'encouragement des sciences et des arts en Canada (Québec, Imprimée par T. Cary \& Co., 1827), 5. 
de littérature. À la Société pour l'encouragement des sciences et des arts, on retrouve la classe littéraire (la littérature, l'histoire, la philologie, la philosophie spéculative, les beaux-arts), la classe du commerce (agriculture, commerce, manufacture, mécanique) et la classe philosophique (l'histoire naturelle, la «chymie», la botanique, la physique, les mathématiques, etc.). Cet effet de spécialisation des disciplines tend à restreindre le terme «littéraire» au sens qui sera donné plus tard à l'épithète «culturel». Il renvoie tout autant à la poésie qu'à la peinture, à la musique et à la scuplture, comme l'indiquent les sujets proposés pour la classe littéraire lors d'un concours ouvert au public en 1827.

\section{Classe littéraire}

1. Une pièce de poésie, dont le sujet sera pris dans le pays, dans quelque genre que ce soit.

2. Un discours sur les avantages d'établir en Canada des institutions scientifiques et littéraires, et sur les moyens de les rendre vraiment utiles et avantageuses au développement des ressources physiques et morales du pays.

3. Un tableau peint à l'huile dont le dessein et la composition seront de l'invention de l'auteur.

4. Un autre à l'eau ou au pastel, avec les mêmes conditions que le précédent.

5. Un spécimen de gravure.

6. Un pièce de musique ${ }^{35}$.

La part du littéraire dans ces sociétés demeure cependant passablement limitée. Au moins les deux tiers des conférences prononcées devant la LHSQ entre 1827 et 1843 portent sur des questions de mathématiques, de physique et d'histoire naturelle, ce qui est peu dire sur le caractère scientifique dont se réclame la société. Cette proportion - qui est à l'image des questions proposées aux concours publics et du contenu de la bibliothèque - est encore plus élévée à la Société médicale de Québec, à la Natural History Society of Montreal et à la Société des artisans de Québec, «où les sujets littéraires ou historiques ne sont pratiquement jamais abordés par les conférenciers ${ }^{36} »$.

35 «Société des sciences et des arts en Canada», La bibliothèque canadienne, 5,5 (octobre 1827): 192.

36 Luc Chartrand, Raymond Duchesne et Yves Gingras, op. cit., 84. 
L'effet de spécialisation des disciplines se poursuit au cours des années 1830 , mais la situation politique qui mène aux insurrections de 1837 et 1838 affecte considérablement la vie des associations qui réduisent leurs activités, voire les interrompent. Qui plus est, la pression des conflits sociaux fait éclater leur caractère bilingue et intégré, tout en favorisant la fondation de sociétés patriotiques rivales.

\section{LES ANNÉES 1830 (LES SOCIÉTÉS PATRIOTIQUES)}

Nous avons vu que le nouveau régime parlementaire de même que le soupçon de l'autorité coloniale amènent les Canadiens à sur-investir dans la seule association politique légitimée qu'ils aient pour défendre leur cause, à savoir la Chambre d'assemblée. Au cours des années 1830, cependant, cet intérêt exclusif est de moins en moins de mise. Les Canadiens prennent alors de plus en plus conscience que, depuis le début du siècle, la nouvelle vision libérale qu'ils proposent n'obtient pas le succès escompté au sein d'une société qui, dans sa très large majorité, est dans l'impossibilité de participer à la compréhension de l'innovation. Certes, les meetings et la presse prolongent sur la place publique les débats de la Chambre. Mais la population, peu scolarisée, n'est pas réellement en mesure de participer activement aux affaires de l'État. Pour y remédier, certains aspirent alors à former des associations qui inciteraient les citoyens à prendre part à l'action collective.

Dans un pays comme le nôtre, où l'éducation n'est pas générale, de semblables réunions seraient le plus puissant levier moral. Elles deviendraient pour nos cultivateurs ce que sont les journaux pour l'habitant des États-Unis. On y viendrait à l'heure du loisir apprendre à juger les actes du gouvernement. Ceux qui dirigent l'opinion publique seraient mieux appréciés, parce qu'ils se trouveraient plus souvent en contact avec leurs concitoyens: la partie pensante de la société acquerrait plus d'influence par une communication plus fréquente avec cette portion, toujours la plus nombreuse, de la société, qui ne peut avoir d'autre éducation que l'instruction auriculaire ${ }^{37}$.

En fait, si les années 1830 sont marquées par la montée du nationalisme qui pousse les principaux éléments ethniques du Canada à fonder leur propre société nationale - les Irlandais lancent la St.Patrick le 17 mars, les Canadiens, la Saint-Jean Baptiste le 24 juin, les Écossais, la St.Andrew le $1^{\text {er }}$ décembre, et les Britanniques, la

37 Le Franc-Parleur, «Des associations et des banques», La Minerve, 8,87 (11 décembre 1834): 1. 
St.George le 19 décembre ${ }^{38}$, - elles le sont bien plus encore par la lutte qui oppose les bureaucrates anglais et les patriotes canadiens. Dans ce contexte, les patriotes prennent conscience de l'importance de former une opinion publique qui puisse faire contrepoids au pouvoir établi. À cet égard, l'exemple des Britanniques leur montre les nombreux avantages à tirer de l'associationnisme.

La Grande Bretagne compte dans chacun de ses villages des sociétés de toutes dénominations; c'est ce qui donne cette uniformité si utile à l'opinion publique; c'est ce qui lui fait cette force morale qu'elle n'a nulle part ailleurs. Détruire les clubs, les associations politiques des Anglais serait changer la forme de leur gouvernement. [...] Si les associations aident à nos adversaires, ils nous aideront de même: et plus à nous qu'à eux, par la raison toute simple que nous sommes les plus nombreux. [...] Dans tous les états libres les associations politiques ont paru les moyens les plus propres à donner à l'opinion publique la prépondérance voulue par le bon sens. Elles existent et se multiplient avec toutes les éventualités du progrès libéral ${ }^{39}$.

Puisque c'est à leurs associations que les Anglais doivent l'efficacité de leur opinion, semble-t-on se dire, les Canadiens ont tout à gagner en les imitant. Aussi est-ce dans ce dessein qu'on préconise des associations politiques et patriotiques, de même que des associations servant à amasser un Fonds de défense nationale. Ce genre d'associations déjà populaire, notamment en Irlande, inspire les Canadiens français qui ne sont pas sans faire la comparaison entre leur situation et celle des Irlandais catholiques. Si l'Association catholique (1823) et l'Institution de la rente catholique ont permis à ces derniers de former un véritable gouvernement parallèle et de se soustraire au joug anglais, pourquoi les Canadiens n'en feraient-ils pas autant?

Nous avons cent fois plus de moyens pécuniaires que les Irlandais souscripteurs au fonds d'O'Connell, estime un correspondant de la Minerve, conséquemment qui d'entre nous ne payerait pas, par exemple, de bon cœur, un chelin par année pour un objet aussi utile et dont le pays retirerait tant d'avantages. Une pareille association, ajoute-t-il, mettrait annuellement plusieurs milliers de louis à notre disposition pour des objets d'intérêt public. L'on pourrait faire publier et répandre dans toutes les

38 Le 15 août 1835, les Français forment à leur tour une société patriotique, «La Société française en Canada», où l'on se réunit chaque année pour célébrer la Saint-Napoléon et entonner des chants nationaux composés pour la circonstance.

39 Le Franc-Parleur, «Des associations et des banques», La Minerve, 8,87 (11 décembre 1834): 1. 
parties du pays et surtout dans les Townships et en Angleterre, des écrits propres à éclairer l'opinion publique ${ }^{40}$.

Pour mener la lutte avec efficacité, il devient donc indispensable aux yeux des patriotes de sensibiliser la population aux enjeux politiques. Le vieux dicton «l'union fait la force» s'impose avec d'autant plus de vigueur que les querelles ethniques sont plus fréquentes.

L'union fait la force, c'est un axiome tellement indubitable que je n'entreprendrai point de vous le prouver; vous en sentez toute l'importance. Il est donc à espérer que chacun de vous voudra bien contribuer, autant qu'il dépendra de lui, à la formation de toutes associations qui peut avoir pour objet de nous réunir et de nous entendre pour déjouer tous les projets de nos ennemis politiques $^{41}$.

Si les appels en faveur des associations se multiplient au cours de la décennie de 1830, ils ne donnent encore que peu de résultats. Aux associations britanniques comme la Quebec Constitutionnal Association (1834), la Montreal Constitutionnal Association (1835), le Doric Club (1836), le Loyal Club Victoria (1837), puis l'Association loyale canadienne (1838), les Canadiens opposent les «Unions patriotiques $^{42} »$, surtout répandues à Montréal à partir de 1835, la Société littéraire $n^{\circ} 1$ (1837), fusionnée bientôt à l'association patriotique les Fils de la liberté ${ }^{43}$, et finalement la société secrète paramilitaire les Frères chasseurs (1838). En fait, plus souvent qu'autrement les regroupements demeurent «informels», et se constituent sporadiquement, parfois même, répression oblige, secrètement, dans les bureaux des imprimeurs de journaux, dans les librairies, les salons, les cafés, et surtout dans les assemblées publiques de citoyens tenues dans différents comtés ${ }^{44}$. Appuyant soit le mouvement patriote soit le mouvement loyaliste, ces groupes investissent les journaux et génèrent des textes qui prennent la forme de résolutions («les 92 Résolutions», «les Résolutions de Saint-Ours»), de discours électoraux, de lettres («Lettre du Comité central aux patriotes»), de manifestes («Adresse des Fils de la liberté de Montréal aux jeunes gens des colonies de l'Amérique du Nord»), de déclarations («Déclaration de l'indépen-

40 Le Pays, «Association pour former un Fonds national pour des objets d'intérêt public», La Minerve, 8,91 (24 décembre 1834): 2.

41 Le Pays, «Nécessité des associations», La Minerve, 8,89 (18 décembre 1834): 1.

42 «Union patriotique», Le Canadien, 5,24 (6 juillet 1835): 1.

43 Voir Le Populaire, 1,62 (30 août 1837): [3].

44 Union des écrivains québécois, Assemblées publiques, résolutions et déclarations de 1837-1838 (Montréal, VLB éditeur, 1988), 304 p., textes recueillis et présentés par Jean-Paul Bernard. 
dance»), de chansons, de narrations et même de poèmes et d'épigrammes.

\author{
Grandement l'on se trompe \\ En pensant les fils de la liberté \\ Tous d'une même trempe. \\ Tous ne sont pas pervers;
}

Le plus grand nombre, c'est la vérité,

N'a seulement que l'esprit de travers ${ }^{45}$.

$\mathrm{Au}$ sein de la plupart de ces associations, la littérature est limitée à un rôle secondaire. Â la Société Aide-toi, le Ciel t'aidera (1834) qui deviendra la Société Saint-Jean-Baptiste, de même qu'à la Société littéraire $\mathrm{n}^{\circ} 1$, on se réunit régulièrement pour lire des essais «sur la littérature ou la politique ${ }^{46} \gg$, mais en raison des problèmes constitutionnels de l'époque, la discussion des questions publiques relègue au deuxième rang, sinon au troisième, les exposés sur la littérature. De plus, si l'on s'en tient au discours des journaux, la littérature n'a souvent d'intérêt que dans la mesure où elle favorise l'acculturation du peuple et la formation d'une opinion publique plus éclairée.

Je suis d'avis que nos meneurs et agitateurs feraient beaucoup mieux dans leurs assemblées de passer des résolutions à l'effet d'organiser et de soutenir des sociétés littéraires et des maisons d'éducation, afin de pouvoir lever le voile de l'ignorance qui couvre les yeux de la masse du peuple, pour qu'il puisse un jour se guider lui-même d'après ses propres connaissances ${ }^{47}$.

Parallèlement à ces visées politiques, commence malgré tout à poindre un discours favorable aux associations professionnelles et plus distinctives. En 1835, la Minerve rapporte que:

En France, presque toute la jeunesse qui suit les professions se réunit en société pour former des espèces d'écoles mutuelles [...]. Les arts libéraux ont suivi cette noble trace. Les littérateurs, les peintres, les artistes forment des groupes d'étudiants, qui travaillent la théorie et la pratique de chacune des branches auxquelles ils se destinent ${ }^{48}$.

En novembre 1838, Napoléon Aubin tient un discours semblable dans le Fantasque. Il préconise un type d'association dans lequel les jeunes

45 [Anonyme], «Épigramme», Le Populaire, 1,88 (30 octobre 1837): 1.

46 Victor Morin, «Clubs et sociétés notoires d'autrefois», Cahiers des Dix, 15 (1950): 187.

47 Un jeune Canadien ami de son pays, Le Populaire, 1,52 (7 août 1837): 2.

48 La Minerve, 9,29 (21 mai 1835): 2. 
trouveraient l'émulation «pour s'aider mutuellement dans leur carrière future»; une association divisible «en cercles particuliers où se fréquenteraient les jeunes gens d'une profession ou d'une classe, d'un métier, d'un quartier et même d'un âge à peu près uniforme ${ }^{49}$ ».

Surtout tenu après 1840 , ce discours mènera à la fondation de plusieurs associations dont entre autres la Société des typographes (1836), la Société musicale de Montréal (1837), les Amateurs typographes (1839), l'Association des notaires de Québec (1840), la Montreal Law Students' Society (1841), la Mercantile Library Association of Montreal (1842), la Société des Amis de Montréal (1844), mais surtout l'Institut canadien de Montréal (1844) et l'Institut canadien de Québec (1848).

\section{LE RÉGIME DE L'UNION}

Après 1840, l'élite libérale canadienne-française prend conscience de l'urgence de se doter de ses propres institutions et associations pour défendre sa nationalité. En vertu de l'Union du Haut et du Bas-Canada en 1840, la Chambre d'assemblée sur laquelle elle fondait tous ses espoirs n'est plus un organisme national, puisque les partis ne sont plus nationaux. Ce ne sont plus des représentants de sociétés antagonistes qui s'y affrontent, mais des équipes de candidats issus de coalitions qui mettent quelque peu dans l'ombre les conflits nationaux antérieurs. Il faut donc trouver un nouveau lieu où la nationalité et la culture canadiennes-françaises puissent se définir et se développer. À l'exemple des Britanniques, on décide alors de se regrouper en associations pour former des bibliothèques et organiser le champ de production intellectuelle.

Jamais avant 1840 cette idée d'association n'a été promue avec autant d'insistance. Aussi les résultats sont-ils à la mesure de la promotion $^{50}$. Au cours des décennies 1840,1850 et 1860 , le phénomène associatif connaît un succès sans précédent, à telle enseigne qu'il entraîne en 1851 la promulgation de la loi des «associations de bibliothèques» qui prévoit des subventions pour les associations qui s'en prévalent. Si les appellations diffèrent d'une association à l'autre (institut canadien, société des amis, cercle littéraire, société de discussion, cabinet de lecture, etc.), la sémantique contemporaine les

49 [Napoléon Aubin], «De la nécessité de former des associations - pour s'amuser», $L e$ Fantasque, 1,40 (5 novembre 1838): 249.

50 Voir à ce sujet les articles d'Yvan Lamonde, «Les associations au Bas-Canada: de nouveaux marchés aux idées (1840-1867)», et «L'association culturelle au Québec au XIX siècle: méthode d'enquête et premiers résultats», Territoires de la culture québécoise (Québec, Presses de l'Université Laval, 1991), 105-115 et 149-179. 
regroupe sous le terme générique d'association «littéraire». Leurs principales fonctions consistent à élaborer, à assimiler et à diffuser les principaux éléments d'un savoir et d'une culture intellectuelle. À cette fin, elles organisent des concours publics, tiennent une bibliothèque et une salle de périodiques, et dispensent un programme de séances publiques comprenant débats entre membres, lecture d'essais et de conférences publiques. En instaurant ainsi, par le cérémonial de séances publiques et la compétition lettrée, le maximum d'ouverture et de publicité, elles cristallisent l'évolution de textes qui, primés ou non, deviendront parfois des livres, des brochures et surtout, grâce à l'affiliation de ces associations avec certains journaux, des articles. L'exemple le plus caractéristique demeure sans conteste celui de l'Institut canadien de Montréal où en plus de trouver une bibliothèque et un musée, plus de 200 débats entre les membres y sont présentés et près de 208 conférences publiques et essais prononcés, dont plusieurs, 94 pour être plus précis, ont été publiés ${ }^{51}$. Signalons également le Cabinet de lecture paroissial ${ }^{52}$ où une dizaine de poèmes, une vingtaine de contes et près de 130 conférences publiques y sont lues de 1857 à 1867 , dont 98 publiées in extenso, ou encore le Cercle littéraire de Montréal où l'on se réunit de 1857 à 1871 pour débattre de questions diverses comme «la Gloire littéraire l'emporte-t-elle sur la gloire militaire?» ou encore «Lequel de l'éloquence, la peinture, la musique et la poésie est l'art le plus important?»

L'apport de ces associations dans la vie littéraire de l'époque est indiscutable. Mais de quel mode d'existence relèvent-elles: celui d'une dépendance à l'endroit d'instances externes au champ littéraire (religion, politique, science, etc.) ou celui d'une autonomie fondée sur des déterminations proprement littéraires?. Dans les associations de Québec, il appert que les belles-lettres s'inscrivent presque uniquement dans le sillage des sciences naturelles et de l'histoire moderne. Un aperçu des activités à la Literary and Historical Society of Quebec de 1827 à 1891 révèle un intérêt marqué pour les questions scientifiques et historiques. Si l'on s'en tient aux chiffres de Ginette Bernatche ${ }^{53}$, sur 374 conférences, $167(44,9 \%)$ traitent de Sciences $(70$ de sciences naturelles), 114 (30,6\%) d'Histoire (66 d'histoire profane) et $26(7 \%)$ ont trait aux belles lettres. De même, à l'Institut canadien de Québec de 1848 à 1914, sur les 274 conférences recensées par

51 Voir à ce sujet, Pierre Rajotte, La pratique de la conférence publique à Montréal (1840-1870), thèse de doctorat, Université Laval, 1991, xxiii-368 p.

52 Marcel Lajeunesse, Les Sulpiciens et la vie culturelle à Montréal au XIX` siècle (Montréal, Fides, 1982), 280 p.

53 Ginette Bernatchez, La Société littéraire et historique de Québec (The Literary and Historical Society of Quebec) 1824-1890, mémoire de maîtrise, Université Laval, 1979, 68-69. 
Daniel Gauvin, l'histoire ressort nettement avec 166 conférences, soit $61 \%$ du corpus global. De ces 166 conférences, la part du lion va surtout à l'histoire moderne, aux mémoires et biographies, et aux voyages et à la géographie avec respectivement 40,35 et 21 mentions. La science occupe également une place importante avec 82 conférences (30\%), alors que «les Belles lettres n'en ont aucune ${ }^{54} »$.

En fait, au moment où sont fondées des associations comme la Société canadienne d'études littéraires et scientifiques (1843), la Société de discussion de Québec (1844?), l'Institut des artisans de Québec (1848), l'Institut canadien de Québec (1848), la Chambre de lecture de Saint Roch (1850), l'Institut catholique de Saint Roch (1852), l'Académie canadienne de Québec (1857), etc., l'histoire, et en particulier l'histoire nationale, connaît une vogue sans précédent au pays. À ce chapitre, la parution de l'Histoire du Canada de FrançoisXavier Garneau et a fortiori la valorisation de la nationalité qu'elle suscita furent une véritable révélation pour la plupart des Canadiens français. Jusqu'alors, ces derniers avaient ignoré presque complètement leur passé et se tenaient pour des vaincus. Pour la première fois, ils se voyaient dans un rôle différent de celui dans lequel on avait bien voulu les confiner. Depuis la Conquête, en effet, les rapports entre Anglais et Canadiens contribuaient à entretenir un sentiment d'humiliation chez les vaincus. Dans les faits, la suprématie de plus en plus absolue des premiers sur le commerce, l'industrie et la haute fonction publique accentuait d'autant le sentiment d'infériorité des seconds. En outre, la seule perception que les Canadiens avaient d'eux-mêmes, de leur histoire, leur venait de certains chroniqueurs anglophones, comme William Smith (History of Canada, 1815), John Fleming (Political Annals of Lower Canada [...], 1828) ou John MacGregor (British America, 1832), qui, plus souvent qu'autrement, confortaient la position des Britanniques. L'échec de la Rébellion de 1837-1838, la répression et qui plus est le rapport Durham, loin de remédier à la situation, la révélaient aux yeux de tous. Or l'Histoire du Canada $(1845,1846,1848,1852)$ de Garneau allait servir d'exutoire et permettre de se défaire d'une véritable tunique de Nessus, l'humiliation collective.

L'impact à Québec de l'Histoire du Canada de Garneau, et un peu plus tard des conférences de l'abbé Jean-Baptiste-Antoine Ferland, a été le ferment d'une véritable mode dans la production littéraire. Au cours de la seconde moitié du XIX ${ }^{\mathrm{e}}$ siècle, les ouvrages, études et

54 Voir Daniel Gauvin, L'Institut canadien de Québec et la vie culturelle à Québec 1848-1914, mémoire de maîtrise, Université Laval, 1984, 161. 
articles qui font référence à l'histoire se multiplient, les uns reprenant la thèse de Garneau, les autres la contestant. La poésie, le roman, le conte, le théâtre, tous les genres littéraires enfin, rappellent chacun à sa façon, les exploits des ancêtres et les desseins spéciaux de la Providence sur la nation canadienne. Les activités déployées au sein des associations de la vieille capitale n'y échappent pas et l'histoire devient un sujet de prédilection.

À Montréal, par contre, dans les décennies 1850 et 1860 en particulier, soit dans le lieu et au moment où il atteint son apogée, le phénomène associatif est largement conditionné par la lutte idéologique qui oppose libéraux et ultramontains ${ }^{55}$. En fait, par le biais des associations, c'est une véritable bataille rangée pour occuper le champ littéraire qui s'engage. Mais cette lutte a ceci de particulier qu'elle met aux prises deux champs institutionnels externes. Avant d'être écrivains en effet, les intervenants sont des partisans libéraux et ultramontains plus soucieux de faire avancer leur cause que de spécifier le littéraire. Si bien que d'un côté comme de l'autre, ce n'est pas tant de la littérature qu'on se préoccupe que de ses effets sur l'opinion publique. Pour les libéraux qui se regroupent à l'Institut canadien de Montréal (1844), la littérature n'a de valeur que dans la mesure où elle éclaire l'opinion et suscite l'esprit critique afin que les citoyens prennent conscience de leurs droits et de la façon de les exercer. Pour les ultramontains en revanche, qui fondent entre autres l'Union catholique (1854), le Cabinet de lecture paroissial (1857) et le Cercle littéraire (1857), il est aussi question d'éclairer les citoyens, mais dans un tout autre sens: celui de les conscientiser sur leurs devoirs et le respect qu'ils doivent à l'ordre établi. Bref, c'est donc dans une perspective visant à se servir de la littérature plutôt que de la servir que, de part et d'autre, on s'associe.

Les nombreux cercles, sociétés ou instituts qui se fondent au cours de la décennie 1840 devraient amener les observateurs à constater que la littérature s'épanouit de préférence dans des milieux restreints. Mais même dans ces lieux privilégiés, la spécification du champ reste ambiguë. Certes on se réunit à l'Institut canadien sous le signe de l'éloquence, mais la littérature n'est pas l'objet d'une prédilection particulière. Dans cette société de débats, tous les sujets sont les bienvenus, ce qui témoigne d'une certaine indétermination, comme le note d'ailleurs fort pertinemment Xavier Marmier en observateur objectif du milieu littéraire canadien: «L'amour des lettres est encore un trait caractéristique de ce pays, un de ses signes de parenté avec l'ancienne

55 Voir à ce sujet, Pierre Rajotte, Les mots du pouvoir ou le pouvoir des mots. Essai d'analyse des stratégies discursives ultramontaines au XIX' siècle (Montréal, l'Hexagone, 1991), 214 p.; voir également «La pratique de la conférence publique à Montréal (1840-1870)», op. cit. 
France. Il y a peu d'écrivains de profession dans le Canada, mais il n'est pas un homme ayant fait, comme on dit, ses humanités, qui ne tienne à rimer son sonnet, d'aiguiser $[$ sic $]$ son madrigal ${ }^{56} \gg$. Marmier parle évidemment d'une élite qui forme un milieu restreint capable à la fois de recevoir des œuvres littéraires parce qu'elle est aussi capable d'en produire. Mais Marmier marque bien l'absence de spécification du champ littéraire car il n'existe aucune distinction entre l'écrivain et le non écrivain, entre le littéraire et le non littéraire. Le sonnet ou le madrigal rimé pour un public de salon n'a pas normalement de prétention à la littérature.

\section{CONCLUSION}

Bien qu'au lendemain de la Conquête, les Britanniques leur fournissent de nombreux modèles d'associations, les Canadiens français sont d'abord peu enclins à y recourir. C'est qu'ils trouvent dans la Chambre d'assemblée à laquelle ils ont accès depuis l'Acte constitutionnel de 1791 le moyen ultime d'établir un rapport de force avec le gouvernement et de défendre leur nationalité. Ce n'est cependant plus le cas après le régime de l'Union en 1840, qui les oblige à trouver un nouveau lieu où leur nationalité puisse se définir et se développer. S'ensuit alors une vogue sans précédent pour les pratiques associatives jusqu'à ce que la loi accordant des subventions aux associations soit abolie vers 1865 .

De ce bref tableau d'un siècle de pratiques associatives, un point apparaît devoir retenir l'attention. On ne peut encore parler d'associations proprement littéraires, mais plutôt d'associations intellectuelles qui intègrent à la fois les discours littéraires, religieux, scientifiques et politiques. Au sein des associations, le terme littéraire est compris dans son sens le plus large et recouvre le champ culturel et scientifique. Si dans un sens restreint on entend par littérature des écrits tels que les poésies, les romans, les pièces de théâtre, dans son usage le plus courant, le terme désigne l'ensemble des connaissances transmissibles par l'écrit. Or si le champ littéraire acquiert de la valeur à l'époque, c'est beaucoup plus à cause de cette dernière acception. En effet, dans la décennie 1840-1850, la mise en place d'un système d'éducation populaire, le perfectionnement de la technique de l'imprimerie, la multiplication des journaux et des bibliothèques permettent ou permettront bientôt à tout citoyen, de quelque classe qu'il soit, d'avoir un libre accès au monde des idées. Dans la mesure où l'éducation, à partir de 1846, devient accessible à tous sans distinction

$56 \mathrm{X}$. Marmier, «Le Canada», Album littéraire et musical de la Minerve, 6,1 (janvier 1851): 16 . 
d'origine, tout est en place en effet pour qu'il se forme un public lecteur d'une importance telle que celui qui le contrôlera aura l'opinion publique pour lui. La question réellement sous-jacente, celle qui valorise le champ littéraire, est de savoir s'il est bon d'encourager une libre circulation des idées ou si plutôt il ne vaut pas mieux les contrôler et les sélectionner. C'est précisément dans le but de défendre l'un ou l'autre de ces points de vue que l'on se regroupe en associations. Conscients des promesses ou des menaces que comporte le passage de l'oral à l'écrit, libéraux et conservateurs comptent en profiter soit pour contester soit pour conforter l'ordre établi. Dans ce contexte, la littérature est soumise à des causes qui lui sont externes et qui, d'une certaine manière, l'empêchent de s'autonomiser. Il en sera ainsi pendant presque tout le $\mathrm{XIX}^{\mathrm{e}}$ siècle. Il faudra attendre la création de l'École littéraire de Montréal en 1895 pour que l'institutionnalisation du champ prenne une configuration moderne.

Ce constat nous amène à formuler une nouvelle hypothèse que nous nous contenterons de poser uniquement puisqu'elle fait l'objet de nos recherches actuelles. Compte tenu du rôle ancillaire que joue la littérature au sein de la plupart des associations «formelles», on peut se demander si les écrivains canadiens n'ont pas préféré les regroupements «informels» et privés, comme par exemple le cercle littéraire de Joseph Quesnel (1798), le salon de Marguerite de Lanaudière tenu à Québec au coin des rues Saint-Louis et Desjardins, celui de LouiseAmélie Panet (1820?) au manoir de Sainte-Mélanie d'Ailleboust et «dans lequel entraient, entre autres, MM. Jacques Viger, D.-B. Viger, le Chevalier d'Estimauville, les deux Stuart, Louis Joseph Papineau, Henri Heney, etc. ${ }^{57} \gg$, le Club des Anciens (1840), l'École patriotique de Québec (1860), groupe réuni dans l'arrière-boutique de la librairie d'Octave Crémazie à Québec, ou cet autre surnommé «le Grenier littéraire» (1875), qui s'assemblait sous les combles de la maison de Louis Fréchette à Montréal. Dans le même ordre d'idée, on peut se demander si des revues littéraires comme la Revue canadienne, la Ruche littéraire, les Soirées canadiennes et le Foyer canadien ne fournissent pas un lieu de regroupement plus spécifique encore à la «création», où les écrivains trouvent le repliement cherché à l'écart des sollicitations politiques ou religieuses. Au demeurant, pour bien cerner les dessous de la vie littéraire au $\mathrm{XIX}^{\mathrm{e}}$ siècle, il importe de déterminer l'ensemble des initiatives qui lui ont permis de se constituer. À cet égard, nous croyons que les regroupements «informels» de lettrés peuvent être révélateurs. C'est précisément dans cette voie encore inexplorée que nous comptons poursuivre nos recherches.

57 La Kermesse, 25 novembre 1892, 132. Il s'agit plutôt ici de Hugues Heney. 